\title{
O Número de Ouro via Método de Newton-Raphson
}

\author{
Djerly Simonetti Viviane Vanessa Dohl Suellen Ribeiro Pardo Garcia* \\ Universidade Tecnológica Federal do Paraná, UTFPR \\ 85902-490, Campus Toledo, Toledo, PR \\ E-mail: adjsimonetti@gmail.com,viviane_dohl@hotmail.com, suellenpardo@yahoo.com.br
}

\section{RESUMO}

O presente trabalho trata de uma abordagem para encontrar uma equação cuja solução se aproxima do número de ouro, pautada na aplicação do método de Newton-Raphson (N-R). Especificamente, busca-se o único valor real positivo para a seguinte equação $x^{2}-x-1=0$. $\mathrm{O}$ encaminhamento para o estudo da aproximação, também se fez por meio do programa $\mathrm{R}$, dado a precisão dos valores, sendo que a escolha do método de N-R deve-se a sua rápida convergência nesse caso.

$\mathrm{O}$ número de ouro representado pela letra grega $\Phi$ (phi), caracteriza-se pela sua autopropagação, ou seja, está presente em diversas áreas do conhecimento [5]. É um número irracional sendo convencional exibi-lo arredondado para três casas decimais: $\Phi=1,618$.

Uma das aplicações de $\Phi$ na geometria é na solução da equação, $f^{2}+f-1=0$, que é obtida do fractal Árvore Áurea. Muitos fractais da natureza possuem como característica principal a ramificação, assim como essa Árvore, alguns desses são obtidos considerando um processo de iteração na qual inicialmente tem-se um ramo de uma unidade de comprimento, donde se obtém dois novos ramos de comprimento $1 / 2$ com $120^{\circ}$, como na Figura 1 . E assim aplicando esse fator de redução $1 / 2$ o processo continua indefinidamente.

Figura 1 - Fractal Árvore.

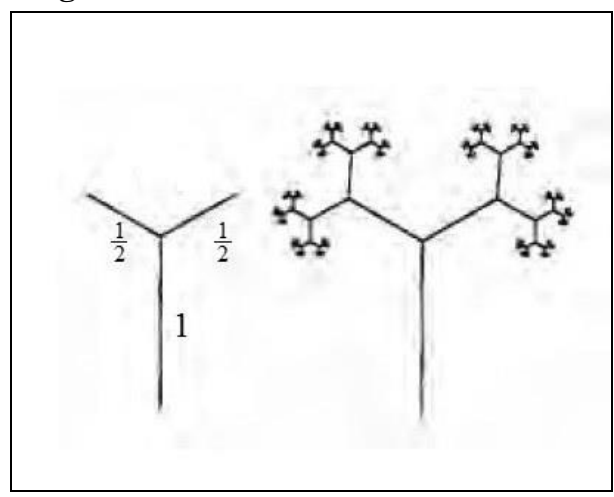

Fonte: Livio, 2002, p. 219.
Figura 2 - Fractal com ramos sobrepostos.

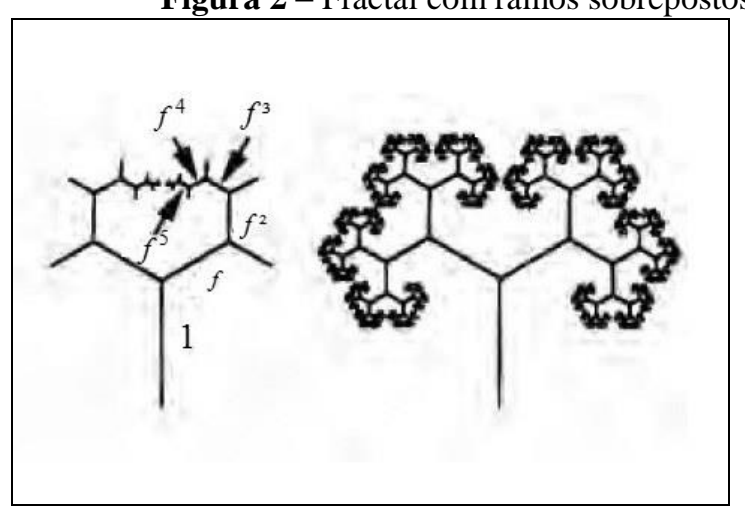

Em sistemas como o sistema circulatório de sangue, pode interessar saber qual o fator de redução que faz com que os ramos comecem a se sobrepor, como na Figura 2. Isto acontece para o fator de redução $\frac{1}{\Phi}=0,618$. A condição para que dois ramos se toquem é que a soma de todos os comprimentos decrescentes dos ramos horizontais a partir de $f^{3}$ é igual ao componente horizontal do ramo de maior comprimento $f$.

Segue que $f \cos 30^{\circ}=f^{3} \cos 30^{\circ}+f^{4} \cos 30^{\circ}+f^{5} \cos 30^{\circ}+f^{6} \cos 30^{\circ}+\ldots$.

Ou seja, $f=f^{3}+f^{4}+f^{5}+f^{6}+\ldots$

\footnotetext{
* Docente da Universidade Tecnológica Federal do Paraná (UTFPR) campus Toledo.
} 
Observa-se do lado direito da equação a soma de uma série geométrica infinita, $\operatorname{logo} f=\frac{f^{3}}{1-f} \Rightarrow 1=\frac{f^{2}}{1-f} \Rightarrow 1-f=f^{2}$. A equação obtida, $f^{2}+f-1=0$, tem única solução real $\frac{\sqrt{5}-1}{2}$, ou seja, $1 / \varphi[4]$.

Note como obter a equação $x^{2}-x-1=0$, cuja solução real positiva é $\Phi$. Considere um segmento $\mathrm{AB}$ de medida $x$ (unidade), estipulando um ponto $\mathrm{C}$ qualquer pertencente ao segmento $\mathrm{AB}$ obtemos dois novos segmentos $\mathrm{AC}$ e $\mathrm{CB}$. $\mathrm{O}$ ponto $\mathrm{C}$ pode ocupar infinitas posições, contudo, só "existe uma única posição - posição de ouro - onde este ponto $\mathrm{C}$ divide o segmento $\mathrm{AB}$ em dois segmentos proporcionais, tal que o quociente entre as medidas do segmento todo pela parte maior é igual ao quociente entre as medidas da parte maior com a parte menor" [1].

Estipulando a medida de AC igual a $a$ e a de CB igual a $x-a$, dado que AB mede $x$, a posição de ouro será obtida de

$$
\frac{x}{a}=\frac{a}{x-a}
$$

Assim, reescreve-se a equação obtida: $x(x-a)=a^{2} \Rightarrow x^{2}-a x-a^{2}=0$. Veja que a solução é $a \frac{(1 \pm \sqrt{5})}{2}$, mas desconsidera-se a solução negativa. O número $\frac{1+\sqrt{5}}{2}$ é o valor de $\Phi$, assim ao fazer $a=1$ temos a equação a qual procuramos.

Nesse contexto, poderíamos determinar a raiz da equação $x^{2}-x-1=0$ por meio da fórmula de Bhaskara também, contudo, nosso objetivo é pautar-se em métodos mais avançados, e como essa equação é polinomial, será de fácil compreensão para o leitor acompanhar o desenvolvimento do método estipulado para o presente texto.

O método de Newton-Raphson (N-R) consiste em um processo iterativo para a determinação de raízes de funções não lineares [1]. Para utilização do método faz-se necessário analisar a função em questão e sua derivada em um determinado ponto, ou seja, se a função apresentar-se simples para derivar, esse método é apropriado.

Para sua aplicação é preciso admitir um chute inicial $x_{i}$ para o valor da raiz procurada, determinando a reta tangente à função nesse ponto $\left[x_{i}, f\left(x_{i}\right)\right]$. Assim o ponto onde essa tangente cruzar o eixo das abcissas é uma estimativa da raiz - Figura 3.

Figura 3 - Descrição gráfica

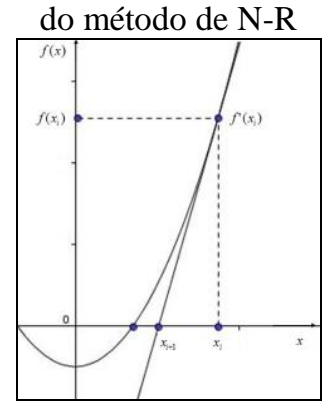

Fonte: Elaborada pelo autor

Observando a interpretação geométrica na Figura 3, pode-se escrever a seguinte equação $f^{\prime}\left(x_{i}\right)=\frac{f\left(x_{i}\right)-0}{x_{i}-x_{i+1}}$. Reorganizando a mesma temos $x_{i+1}=x_{i}-\frac{f\left(x_{i}\right)}{f^{\prime}\left(x_{i}\right)}$, a qual é chamada de fórmula de Newton-Raphson [3].

Para que possamos aplicar o método, se faz necessário verificar a convergência do mesmo, para poder prosseguir com os cálculos. Para tanto, considere o teorema: seja $f \in[a, b]$. Se $x \in[a, b]$ tal que $f(x)=0$ e $f^{\prime}(x) \neq 0$, então existe um $\delta>0$, tal que o método de N-R gera 
uma sequência $\left\{x_{n}\right\}_{n=0}^{\infty}$ que converge para $x$ para qualquer aproximação inicial $x_{0} \in[x-\delta, x+\delta]^{1}[2]$. Como a $f(x)=x^{2}-x-1$, a convergência está garantida.

O método de Newton-Raphson é também um método iterativo assim como a maioria dos métodos numéricos, para isso é necessário estipular um erro máximo que determinará o critério de parada das iterações a serem realizadas. O erro $(\varepsilon)$ é dado calculando a $f$ do valor encontrado na iteração, e esse valor deverá ser menor que o erro desejado para que se encerre o processo de iterações.

O chute inicial é dado de forma que a convergência seja mais rápida e pode ser dado com base no gráfico da equação, no entanto se o chute for muito distante da raiz, a quantidade de iterações será muito maior.

Na equação onde a solução é o número de ouro temos $f\left(x_{i}\right)=x^{2}-x-1 \Rightarrow f^{\prime}\left(x_{i}\right)=2 x-1$. Admitindo um erro de aproximação para essa raiz de 0,0000001e um chute inicial para $x_{i}$ de 1,2 temos

$$
x_{i+1}=1,2-\frac{(1,2)^{2}-1,2-1}{2(1,2)-1}=1,742857142
$$

Calculando o erro $(\varepsilon)$, obtemos $f(1,742857143)=0,294693878$, o qual é maior do que o erro permitido, e portanto é necessário continuar com as iterações. Assim, admitimos 1,742857143 para $x_{i}$, para continuar as iterações. Resumidamente obtermos o seguinte:

Tabela 1: resultados das iterações

\begin{tabular}{c|c|c}
\hline$x_{i}$ & $x_{i+1}$ & $\varepsilon$ \\
\hline 1,2 & 1,742857143 & 0,294693878 \\
1,742857143 & 1,624302135 & 0,014055290 \\
1,624302135 & 1,618051462 & 0,000390710 \\
1,618051462 & 1,618033989 & 0,0000000001 \\
\hline
\end{tabular}

Portanto, a solução aproximada para a equação é 1,618033989. Em comparação, obtém-se a solução pelo software R, o qual apresenta como resultado 1,618034.

O método de Newton Raphson é eficiente para encontrar uma aproximação da raiz da equação $x^{2}-x-1=0$. Mediante a proposta apresentada, observa-se a convergência para o valor aproximado do número de ouro. Lembrando que, uma das características marcantes do método de N-R em relação a outros métodos numéricos é acelerar o processo de convergência, ou seja, menor número de iterações.

Palavras-chave: Número de Ouro, método de Newton Raphson, Árvore Áurea

\section{Referências}

[1] BIEMBEnGUT, Maria Salett. e HEIN, Nelson. Modelagem Matemática no Ensino. - $3^{\mathrm{a}}$ ed. - São Paulo: Contexto, 2003.

[2] BURDEN, Richard L. e FAIRES, J. Douglas. Análise Numérica. - $8^{\mathrm{a}}$ ed. - São Paulo: Cengage Learning, 2008.

[3] CHAPRA, Steven C. e CANALE, Raymond P. Métodos Numéricos para engenharia. - $5^{\mathrm{a}}$ ed. - São Paulo, McGraw-Hill, 2008.

[4] LIVIO, Mario. The Golden Ratio: the story of Phi, the world's most astonishing number. New York: Broadway Books, 2002.

[5] STEWART, Ian, Almanaque das curiosidades matemáticas. - Rio de Janeiro: Zahar, 2009.

\footnotetext{
${ }^{1}$ Para um maior aprofundamento da demonstração pode-se ler Análise Numérica, de Richard L. Burden e J. Douglas Faires, 2008.
} 